\title{
IMPULSIVITY OR SUB-OPTIMAL REWARD MAXIMIZATION in Delay Discounting? A Critical Discussion
}

\author{
Espen A. Sjoberg \& Espen B. Johansen \\ Department of Behavioral Sciences, Oslo Metropolitan University, Oslo, Norway. \\ espen.sjoberg@oslomet.no
}

\begin{abstract}
The delay discounting paradigm involves choosing between a small, immediate reward and a larger, delayed reward. As the delay between response and reinforcer increases for the large reward, people with Attention-Deficit/Hyperactivity Disorder (ADHD) tend to choose the small reward more often than controls. Studies on an animal model of ADHD, the Spontaneously Hypertensive Rat (SHR), find similar results. This pattern is typically interpreted as impulsive behaviour, implying that an unwillingness to wait decreases the likelihood of choosing the large reward. Alternatively, the results can be interpreted in terms of optimality, where a switch in preference from large to small rewards indicates sub-optimal behaviour. We critically discuss available evidence on delay discounting in ADHD and the SHR model, and evaluate whether an optimality perspective provides a useful interpretation of the data. Our findings suggest that optimality is a term that contributes little to explaining behaviour in delay discounting, outside of acting as a mathematical measure of reward maximization. Impulsive behaviour is best explained as a consequence of controlling variables, first and foremost the delay between response and reward, but also to a certain degree the intertrial-interval and the reward magnitude.
\end{abstract}

Keywords: Impulsivity, Optimality Theory, Attention-Deficit/Hyperactivity Disorder, Spontaneously Hypertensive Rat, Inter-trial-interval. 


\section{INTRODUCTION}

Impulsivity is a multi-dimensional concept that evokes immediate recognition, but has a variety of definitions (Dalley, Everitt, \& Robbins, 2011). Generally, human impulsivity is described as the tendency to act prematurely and without foresight. We make a sudden purchase that we later regret because the next week the same item was on sale. We impatiently interrupt someone because we were itching to speak. These are instances we all recognize either in ourselves or in others, and are acts that we can label as impulsive behaviours. Impulsivity has been observed in a variety of forms across many different species, with multiple theories and mathematical equations proposed to best explain the behaviour (Fox, Hand, \& Reilly, 2008; Killeen, 2015; Marco et al., 2009; Odum, 2011; Rosati, Stevens, Hare, \& Hauser, 2007; Sonuga-Barke, Taylor, Sembi, \& Smith, 1992; Wolff \& Leander, 2002).

Impulsivity is especially important in research on Attention-Deficit/Hyperactivity Disorder (ADHD), as patients are typically classified into one of three categories: hyperactive-impulsive, inattentive, or both (American Psychiatric Association, 2013). The DSM-5 describes impulsive symptoms as blurting out answers before the question has been completed, difficulty waiting turn, and interruption and intrusion on others. Multiple theories exist on ADHD impulsivity (Sagvolden, Johansen, Aase, \& Russell, 2005; Sonuga-Barke et al., 1992), with arguably the most common method for evaluating impulsive behaviour being the delay discounting procedure (Mazur, 1987). This article critically reviews this paradigm within the context of optimality, and whether behaviours classified as "impulsive" could alternatively be interpreted as "sub-optimal" behaviour.

\section{DELAY DISCOUNTING}

Delay discounting is a method used to study a participant's devaluation of a reward as a function of time. The approach in its simplest form is straightforward: The participant must choose between a small reward that arrives immediately and a larger reward that arrives after a delay. For example, the choice between $\$ 1$ now and $\$ 2$ in twenty seconds. When making this choice, the individual makes a subjective evaluation of the cost of time, and judges whether the extra wait is worth the extra reward. Two dollars may be twice the amount as $\$ 1$, but the presence of a delay element between choice and receiving the reward devalues the amount. In an experiment, these variables are manipulated in order to assess their impact, for example by expanding the ratio between small vs large rewards (e.g. \$1 vs. \$5) or systematically increasing the delay between choice and delivery of the large reward (e.g. waiting five seconds vs. one minute).

In a typical delay discounting study, the small and immediate reward is labelled SS ("small sooner") while the delayed, larger reward is called LL ("large later"). Participants are usually exposed to the choice paradigm multiple times during an experiment, with an inter-trial-interval between the trials (Fig. 1). In general, the term "delay" refers to the time between choice and delivery of the large reward, LL. Initially, the delay for LL is 
absent or short, and naturally the participant therefore prefers the large reward, e.g. the choice between obtaining $\$ 1$ or $\$ 2$ immediately is fairly obvious. The delay for LL is then manipulated to the point where a switch in preference is eventually observed. That is, at some point the smaller reward is subjectively deemed more valuable to the participant compared to the larger reward. If this preference switch happens earlier in one group compared to another, the first group is said to be impulsive.

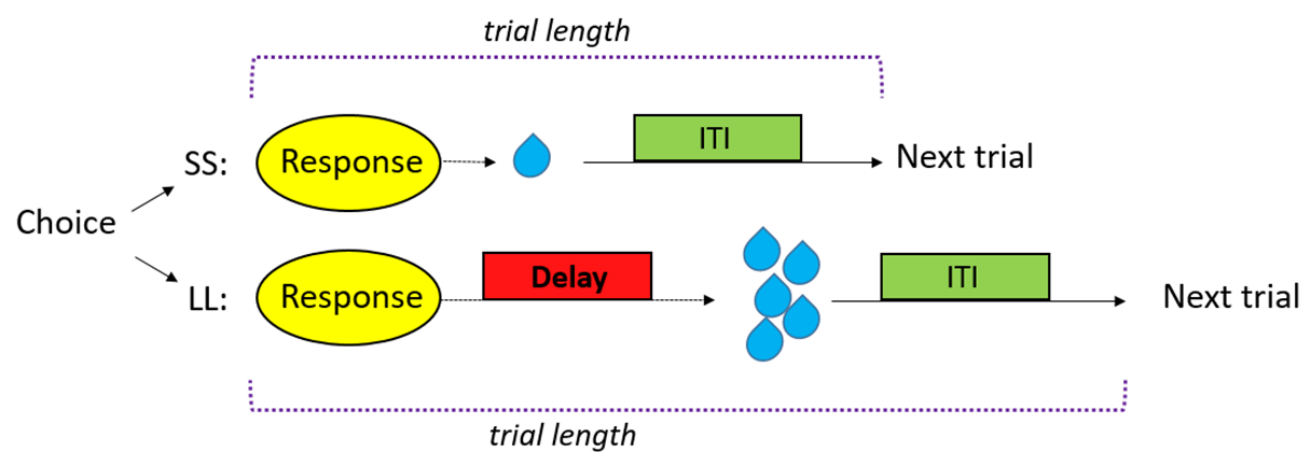

Figure 1: A standard delay discounting setup. The participant can choose between a small reward immediately and a larger reward that arrives after a specific delay. This version can also be called unbalanced because the trial is not of equal length for the two choices.

The reward used in the experiments varies, but for people with ADHD money (Scheres, Lee, \& Sumiya, 2008), sweets (Dalen, Sonuga-Barke, Hall, \& Remington, 2004), or points (Marco et al., 2009) have been used. Food pellets (Fox et al., 2008) or water drops (Reynolds, De Wit, \& Richards, 2002) are typically used for rats, with grain for pigeons (Mazur, 1987; Smethells \& Reilly, 2015). In animal studies, the term used for consequences of behaviour that select, increase and control behaviour is usually reinforcer, which is a strictly behavioural term defined by its effect, and with no reference to subjective or cognitive states. The overlapping term reward connotes "reinforcer" as well as subjective states like "pleasure" and "incentive", and has an assumed (e.g. points, money) rather than empirically shown effect on behaviour (Robbins \& Everitt, 1996; White, 1989). For simplicity, however, and to keep in line with the concepts used in the ADHD studies, we will use the terms reinforcer and reward synonymously throughout the article.

Delay discounting has been researched extensively since the paradigm was suggested in its first form by James Mazur in 1987, particularly in recent years. In a special issue on delay discounting in the Journal of Experimental Analysis of Behaviour, it was found that the amount of published articles on delay discounting per year had increased dramatically from just a few in 2000 to almost 200 in 2014 (Bickel, MacKillop, Madden, Odum, \& Yi, 2015). 


\section{IMPULSIVENESS IN ADHD - DELAY DISCOUNTING AND DELAY AVERSION}

In people with ADHD (usually children), the delay discounting paradigm has been employed in order to determine which variables contribute to impulsive behaviour. One central study is by Sonuga-Barke et al. (1992), who had participants play a game where they could choose between receiving one point after a 2 -second delay or two points after 30 seconds.

In one condition, but not the other, the reward delivery was followed by a post reward delay (henceforth referred to as the inter-trial-interval). Importantly, in this postdelay setup, the length of the inter-trial-interval that followed reward delivery was adjusted in accordance with the delay for LL. Recall that participants could choose between a small reward in two seconds or a larger reward in 30 seconds. Thus, the intertrial-interval for the LL option was 2 seconds, while for the SS option it was 30 seconds. This ensures that the trial length was always 32 seconds, regardless of choice. Such a setup can be called a compensating setup (Fig. 2). In the no-post-delay setup, this intertrial-interval was absent. Because the trial length of LL choices now become longer compared to SS, this is what we will refer to as an unbalanced setup (Fig. 1).

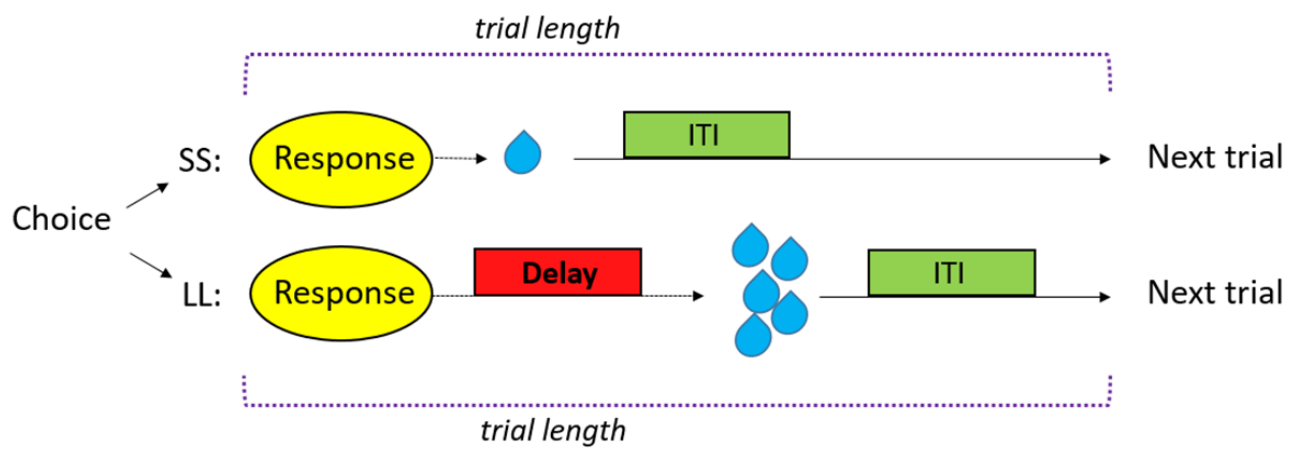

Figure 2: A compensating (or balanced) design, where the inter-trial-interval for each choice is adjusted in accordance with the delay of the opposite choice. This way, the trial length is always the same regardless of choice.

An important note regarding Sonuga-Barke et al. (1992) study is that SS was the most economic choice in the unbalanced setup. This was because choosing SS awarded one point per two seconds, compared to two points per 30 seconds for the LL. Thus, SS allowed for a greater amount of rewards to be obtained during the experimental session. However, in the compensating setup the trial lengths were always the same, effectively cancelling out all variables from an optimization viewpoint except the size of the reward. Therefore, in the compensating setup, LL is the optimal choice and will produce more rewards. In their study, both ADHD children and controls seemed to follow a rewardmaximization, or optimality-based, strategy, preferring LL in the compensating setup and SS in the unbalanced setup. 
In a follow-up, Sonuga-Barke et al. (1992) used an unbalanced setup without an intertrial-interval where the session ended either after 20 trials or after 10 minutes ("Trial" and "Time" conditions, respectively). In the Trial condition, the LL produced the highest amount of rewards because the session had a restricted number of choices available. However, choosing LL also involved longer waiting times before the session ended. By contrast, in the Time condition the session always ended after 10 minutes, and so choosing SS would produce more rewards, effectively one reward every two seconds (SS) compared to one reward every 15 seconds $(\mathrm{LL}-30 \mathrm{~s} / 2=15)$.

Interestingly, $\mathrm{ADHD}$ children preferred SS in both conditions, while controls only preferred SS in the Time condition. The interpretation of this finding was that children with $\mathrm{ADHD}$ were unwilling to endure the length of the trial in order to get a larger reward. Sonuga-Barke et al. (1992) therefore suggested that ADHD children are delay averse. Despite the name, this theory does not suggest that impulsive behaviour is governed by delays between choice and reward, but rather that impulsivity is the result of a tendency to make choices that reduce overall trial length, or overall waiting time. In other words, what matters is how much time a person has to invest as a whole for each choice. A later replication found similar results, but concluded that both the total trial length and the time between response and reward contribute to the choices made (Marco et al., 2009). This combined approach is called the dual component model of ADHD (although one could argue this would be multicollinearity).

\section{CHOICE: REWARD MAXIMIZING?}

Another way to interpret the results of Sonuga-Barke et al. (1992) is to say that the children with ADHD acted sub-optimally in the Trials condition. By "sub-optimal" we here refer to choices not maximizing the number of obtained rewards. That is, the choice to switch to SS was not a result of impatience, but rather a reasoning error regarding variables that produce the highest amount of rewards. Killeen (2015) has called this a capitalist view of $\mathrm{ADHD}$, and argues that such an interpretation assumes that reward magnitude is valued equally for all participants. This is a risky assumption to make, because people's estimation of how valuable a reward is to them will depend on both prior experience (e.g. conditioned rewards like money, points) and current state, e.g. deprivation, satiation (Eisenberger \& Masterson, 1986). In addition, small delayed rewards are discounted more steeply than large delayed rewards (Green, Myerson, \& Ostaszewski, 1999). That is, the perceived value of a small reward is reduced more profoundly than a larger reward when a delay is introduced. In animal studies, where the organism is typically deprived of the reward prior to the experiment, the SS may be more appealing because the LL reward magnitude is largely irrelevant: the animal is hungry and wants whichever option gives it food immediately, irrespective of what is optimal in the long run.

This presents a complicated problem: while it is certainly mathematically possible to assert which choice produces the maximum amount of rewards, is it also fair to say that this choice is "optimal"? One could argue that organisms should always strive to attain the highest amount of rewards possible, but in practice this may not be so simple. In 
delay discounting procedures, reward maximizing depends on several variables and parameters. In an unbalanced design, LL choices will always maximize reward if testing is terminated after a fixed number of trials. If testing is terminated after a fixed time duration, then reward maximizing depends on the SS/LL reward magnitude ratio and the SS/LL trial length ratio (i.e. how much more valuable is the LL choice in the long run: Sjoberg, Holth \& Johansen, 2016). The experimenter can easily calculate the reward maximizing, or "optimal", choice, and human participants are often informed about the experimental parameters beforehand and can make a reasonable mental assessment of what choice produces the most rewards. Still, humans may choose $50 \$$ today over $55 \$$ in ten days. This choice is not easily explained as a reasoning error regarding what choice produces the highest amount of rewards. Choices in humans as well as other animals may not conform to mathematical reward-maximizing, but are influenced by a number of additional variables.

In animal experiments, we cannot instruct the animals beforehand, and even with extensive training it is risky to assume that the animal fully "understands" the parameters operating during the session (Holth, 2001). With animals, it is better to adopt an ethological approach and describe the observed behaviour within the context of the experimental variables rather than making any assumptions about the animals' capacity to mathematically master the paradigm. This also means that the behaviour of human subjects may be governed by the instructions given at the start of the study, and not necessarily affected by reward and delay to the degree assumed. This makes a clear interpretation of data more difficult as group differences in observed choices may reflect a range of factors including e.g. instruction (Alessandri \& Cançado, 2017; Matthews, Shimoff, Catania, \& Sagvolden, 1977), slowed learning (Cournoyer \& Trudel, 1991), and altered sensitivity to reward rates (Tripp \& Alsop, 1999; but see Luman, Van Mee, Oosterlaan, Sergeant, \& Geurts, 2009). An individual's choice is also not static and can change with time, e.g. an individual may choose to save money a week before payday, but choose to spend the money on clothes when the paycheck arrives (Ainslie, 1975; Rachlin, 1974). Thus, a small immediate reinforcer may outcompete a large delayed reinforcer if the time of choice takes place close in time to the small immediate reinforcer.

Furthermore, choice may be controlled by reward maximizing expressed as the total number of obtained rewards at the completion of testing (molar view) or as the maximum rewards per minute (molecular view). In the molecular view, the organism will select whichever alternative has the highest value at that moment, an approach that could also be called Momentary Maximization Theory. Thus, the "optimal" choice becomes a matter of subjective perspective on the task: to what degree do the participants perceive the impact of the relevant variables, and are their choices based on long- or short-term goals? The optimal choice changes depending on the goal of the participant, and the degree of comprehension of the experimental variables by the participant may in turn affect his or her goal. Note that "comprehension", inferred from observations of choice, is seldom measured in human studies and almost never in animal studies. We can argue that an organism "understands" or "comprehends" a variable with an observed effect on choice, but the inverse, that variables not affecting choice are not "comprehended", is not a valid conclusion. Thus, references to "comprehension" or 
"understanding" is largely redundant; all we can conclude with confidence is whether variables affect choice or not (Holth, 2001). All of these aspects illustrate how explaining impulsive behaviour is a complicated endeavour.

\section{OPTIMAL, IMPULSIVE, OR BOTH?}

When we interpret the behavioural data in mathematical ways, it raises the question of whether we should interpret the behaviour as impulsive or sub-optimal decision making. Sonuga-Barke et al. (1992) concluded that children with ADHD are delay averse rather than reward maximizers since they acted optimal in the compensating setup. However, the two approaches are not mutually exclusive, and the answer could be both: acting impulsive could be considered a sub-optimal behaviour. As long as the LL can produce more rewards through repeated trials, switching to SS will always be a sub-optimal choice from a mathematical viewpoint, but what we are really interested in is why this switch occurs. We cannot say it occurs because the subject is impulsive: impulsivity is an interpretation of the data, not its cause. It might be better to say that the tendency for a subject to make sub-optimal delay discounting choices may be termed "impulsivity". We then ask what variables control this behaviour, e.g. the response-delay interval or the inter-trial-interval.

The most general model of choice, optimality theory, deals with foraging behaviour and choices in a natural setting, and attempts to describe the optimal choice for any situation by weighting many variables in a cost-benefit perspective (MacArthur \& Pianka, 1966). A related model of foraging describes energy gain over time by dividing the size of the reward by the delay before it is received, which effectively gives an index of rewards per second for each response, or EoR: expectation of rates (Bateson \& Kacelnik, 1996). An expansion of the EoR equation involves calculating the relative weight of LL compared to SS for each choice trial, based on ratios of reward magnitude, delay length, and inter-trial-interval lengths, in order to get a measure of the long-term value for choosing the LL alternative (Sjoberg et al., 2016). Another approach takes into account perception of time, and describes reward discounting as the actual reward value divided by the sum of pre- and post-reward delays (Blanchard, Pearson, \& Hayden, 2013). This latter equation recognizes that an animal's time estimate is affected by learning and attention, and therefore the perceived value of each choice will vary from individual to individual (Hayden, 2016).

Furthermore, choice can be viewed as either dichotomous or continuous. A dichotomous viewpoint indicates an exclusive preference for one alternative over another, while a continuous viewpoint refers to degrees of preference. Therefore, impulsivity and delay discounting data should perhaps be looked at in terms of preference switching, i.e. the crossover point where the manipulated conditions make e.g. the LL appear unfavourable and the organism switches preference from the LL to the SS. The question of whether choice and preference is best regarded as a dichotomous or a continuous variable is best answered by data, and may depend on methods of testing. Of importance for interpreting ADHD data, however, is that preference (whether exclusive 
or not) is established through repeated exposure. Testing in ADHD studies is often limited, and group differences could reflect slowed learning of choice consequences that may change with repeated testing rather than real differences in preference. Here, data from animal model studies can be valuable.

Thus, there are multiple ways to explain and predict choice patterns during delay discounting, but this also makes it difficult to assert whether the observed behaviour is optimal or not. Ultimately, it depends on how we define "optimality": does it refer to reward-maximization, or to what an organism perceives to be the most favourable outcome, all factors considered? Even if we conclude that optimal behaviour is reward maximizing, then there are multiple approaches on how to calculate the optimal choice. This suggests that talking about optimal behaviour is a cul-de-sac, and works best as a mathematical assessment of the experimental methodology rather than an explanation of behaviour.

\section{THE MANY ASSUMPTIONS OF ANIMAL KNOWLEDGE IN ANIMAL EXPERIMENTS}

When conducting delay discounting experiments on animals, we ultimately cannot ascertain to what degree the animals truly "understand" the variables related to their choice. This makes any calculation of optimality difficult, because we need to map out all the relevant variables, the relative weight of each of the variables, and their possible interactions. If an animal's choice is uninfluenced by e.g. the inter-trial-interval or reward size, how can a mathematically "optimal" choice be calculated?

To illustrate the various issues with animal delay discounting, we will primarily use the Spontaneously Hypertensive Rat (SHR), an animal model of ADHD. Both SHRs and people with ADHD show signs of inattention (Sagvolden, 2000; Sagvolden, Aase, Zeiner, \& Berger, 1998), impulsivity (Fox et al., 2008; Malloy-Diniz, Fuentes, Leite, Correa, \& Bechara, 2007), hyperactivity (Berger \& Sagvolden, 1998; Sagvolden et al., 1998 ) and behavioural variability (Johansen, Killeen, \& Sagvolden, 2007; Scheres et al., 2008).

\section{Preference test}

In a rat experiment on delay discounting, the training and testing process is unavoidably longer than with humans. Prior to the experimental phase, the rats have to habituate to their testing environment, be trained to respond, and ideally pass a preference test to ensure that the rats prefer LL when no delays are present for either choice. The consideration of these issues underlines how the construct validity of an animal model study is challenged before the experiment even begins (Sjoberg, 2017).

Before animals can be tested in delay discounting, a preference test must be conducted, because it is important to ensure that the animals express a preference for the large reward prior to any manipulations. Otherwise, we are simply assuming that the animals have an intuitive preference for the large reward and that they will prefer it once they have identified which response produces it. A standard criterion is that an animal 
needs to show a $66 \%$ preference for LL before any experimental manipulation begins. Strangely, some SHR studies do not make any mention of a preference test prior to the experiment (Hand, Fox, \& Reilly, 2009; Orduña, 2015; Sutherland et al., 2009; Wooters \& Bardo, 2011). Others used a test, but the details (particularly regarding the criterion) are unclear (Adriani, Caprioli, Granstrem, Carli, \& Laviola, 2003; Botanas et al., 2016; Fox et al., 2008; Ibias \& Pellón, 2011). Sometimes a preference test was used, but included a delay component, a variable that ideally should not be introduced until the experimental phase begins (Bizot et al., 2007; Garcia \& Kirkpatrick, 2013; Pardey, Homewood, Taylor, \& Cornish, 2009). Other studies explicitly reported the details of a preference test and used no delays (Adriani et al., 2004; Íbias \& Pellón, 2014; Sundbø, 2013). The issue with these findings is that comparison of data across SHR studies is difficult, because the various researchers have made different assumptions regarding the rats' preference for the large reward. Before we can say anything regarding impulsive behaviour in rats, we first have to establish that they prefer the large reward in the absence of any delay variable.

\section{The delay between response and reward}

Once the training phase is complete, most SHR studies follow one of the two setups described earlier: compensating or unbalanced, with the only difference being that in an unbalanced setup the choices are not of equal trial length. In a compensating design, the argument is that trial length (delay aversion) is controlled for, and the independent variable is the time between response and reward. SHRs still express a switch in preference sooner than controls in this setup, suggesting that delay aversion is not a good explanation for their impulsive behaviour (Fox et al., 2008; Hand et al., 2009). Instead, the delay between response and reward delivery appears to be the main controlling variable.

A problem with the compensating design, however, is that we cannot be certain if the contributing variable to a change in behaviour was the size of LL, the addition of the delay, the reduction of the inter-trial-interval, or possible interactions between these variables. While the delay between response and reward is a likely explanation, the intertrial-interval is reduced every time delay increases, meaning that the two variables cannot be separated, and therefore we cannot be certain to what degree they control behaviour. The size of LL has also been found to impact impulsive choice (Garcia \& Kirkpatrick, 2013), but one could argue this is controlled for in a compensating setup.

\section{The problematic inter-trial-interval}

Another problem with the compensating delay discounting design is the assumption that the animals are affected by consequences following reward delivery (Hayden, 2016). In other words, it assumes that the animals are aware of a waiting time between trials. If the animals ignore the inter-trial-interval, the consequence is that a compensating design becomes effectively identical to an unbalanced design, where the only two variables of importance are the size of the reward and the delay between response and reward delivery. 
Several animal studies on delay discounting have found that the length of the intertrial-interval has little or no effect on choice, at least compared to the delay component. This is shown in pigeons (Goldshmidt, Lattal, \& Fantino, 1998), starlings (Bateson \& Kacelnik, 1996), monkeys (Pearson, Hayden, \& Platt, 2010) and rats (Sjoberg, Holth, Ottåsen, \& Johansen, 2017). A possible explanation for this is an ecological one: In nature, the length of the inter-trial-interval is likely to correlate with the size of the reward, since the animal needs to consume and digest. The inter-trial-interval would not become shorter as LL grows larger (Hayden, 2016). We may use the analogy of hunting prey: An increased delay is analogous to increased hunting or searching time, which may secure a larger prey (LL reward). As predicted by foraging theory, many animals express a correlation between searching time and prey size (Elner \& Hughes, 1978; Werner \& Hall, 1974), and that degree of hunger predicts likelihood of predation (Jeschke, Kopp, $\&$ Tollrian, 2002). This would, in turn, lead to a longer time until the next hunt due to consummation and digestion time (inter-trial-interval). It would certainly not lead to a shorter inter-trial-interval, which is what the compensating delay discounting setup achieves. Therefore, employing the compensating design in animal testing reduces its ecological validity.

Furthermore, there are few situations in nature where a food choice continuously repeats. Animals do not make a choice between different food amounts, only to have the same choice available again almost immediately. It may move to a new foraging patch and be exposed to similar dilemmas, but here the choices are found by the animal, rather than presented to it continuously. There are a few exceptions to this rule, e.g. hummingbirds can return to the same flower within a certain time interval (Pyke, 1978). An animal may also return to the same foraging patch during the next migratory season (Strandberg, Klaassen, Hake, Olofsson, \& Alerstam, 2009), but such comparisons to the delay discounting experiment has low validity due to the length of the temporal variables involved (Sjoberg, 2017).

The inter-trial-interval appears to have minimal impact on choice if the delay between response and reward for LL is absent or short (Sjoberg et al., 2017; Smethells \& Reilly, 2015). However, if the delay is long (approx. six seconds or more), then short inter-trial-intervals results in impulsive choices, while long ones do not (Smethells \& Reilly, 2015). This suggests that the delay and inter-trial-interval components interacts. In an experiment with monkeys, Blanchard et al. (2013) concluded that the animals appeared to be aware of the presence of an inter-trial-interval, but greatly underestimated its length unless a visual cue was provided to indicate its duration. This suggests that the inter-trial-interval does have an effect, but only if the animals are able to determine its length. Choice, in this set-up and the chosen parameters, appears to be controlled to a greater degree by the delay variable than by the inter-trial-interval. The concept of the delay-of-reinforcement gradient may help to explain this effect: Here, a response is successfully reinforced as long as its consequences happen within a certain timeframe (Sagvolden et al., 2005). If the consequence happens too late, the reinforcer loses its potency, and the response is not strengthened or maintained. The inter-trial-interval has no reinforcing component, and here the animals do not pay as much attention to it as they would the delay component. Adding a reward after the inter-trial-interval increases its salience, and therefore its impact on the discounting (Blanchard et al., 2013). 
These issues illustrate that multiple variables impact impulsive choice, and that these variables appear to hold different weight. Weighing these variables in terms of their contribution is possible, but such actions also require that interaction effects are taken into account. Additionally, such weighting is somewhat speculative, and it means we are moving into a muddy domain where we tweak our equations post-hoc until we force them to make sense, rendering anything optimal (Pierce \& Ollason, 1987). It is therefore incredibly difficult to talk about optimal choices, as the actual choice is context-specific for any given set of parameters.

\section{SUMMARY AND CONCLUSION}

While an optimality calculation is possible to conduct in delay discounting, it is wrought with complications and assumptions, and its validity can be questioned. What is "optimal" depends on too many variables, both experimental and contextual, and the term is best reserved to be used as a description of reward-maximization, and not to describe behavior. It is, perhaps, best to regard the impulsive behaviour of the rats as a product of the experimental variables present, in particular the delay between response and reward. This appears to be the most reliable variable in terms of predicting choice behaviour. In humans, other variables can affect the outcome, as seen with the dualcomponent theory of ADHD. Humans are able to follow instructions and can be primed and told of the parameters of an experiment before participating in it. Animal studies, on the other hand, require pre-training, an accommodating setup and usually prolonged testing, but here we also have greater experimental control over the learning history and variables influencing choice.

It is clear that multiple variables govern the behaviours of rats in the delay discounting paradigm, such as delay, inter-trial-interval, and reward size. These variables carry different discounting weight, with the delay component seemingly being the strongest predictor, but the variables most likely also interact. In terms of optimality, it becomes a question of determining exactly to what degree these variables contribute to determining choice, and in animal models of ADHD whether this contribution is different in SHRs than in controls. A precise answer to this question is difficult to achieve, and an adequate equation would have to take into account all of these variables, their weight, their interactions, as well as how the animals perceive time. For these reasons, it may be more prudent to talk about impulsivity in delay discounting in terms of contributing variables, rather than as a discussion on optimality, aside from the observation that a switch in preference is considered sub-optimal. Optimality, then, is not a useful term outside of a mathematical construct that outlines the choice that produces the highest amount of rewards. It would be naïve to expect organisms to act as supercomputers, carefully evaluating all the experimental variables, and then calling their behaviour sub-optimal when it deviates from what is mathematically the logical choice. 


\section{ACKNOWLEDGEMENTS}

Thank you to Raquel G. Wilner for proofreading and making multiple grammatical corrections and suggestions. Thanks to Elisabeth Oberzaucher for giving us an extended deadline to finish the original submission.

\section{REFERENCES}

Adriani, W., Caprioli, A., Granstrem, O., Carli, M., \& Laviola, G. (2003). The spontaneously hypertensive-rat as an animal model of ADHD: evidence for impulsive and nonimpulsive subpopulations. Neuroscience and Biobehavioral Reviews, 27(7), 639-651. DOI

Adriani, W., Rea, M., Baviera, M., Invernizzi, W., Carli, M., Ghirardi, O., Caprioli, A., \& Laviola, G. (2004). Acetyl-L-carnitine reduces impulsive behaviour in adolescent rats. Psychopharmacology, 176(3-4), 296-304. DOI

Ainslie, G. (1975). Specious reward: a behavioral theory of impulsiveness and impulse control. Psychological bulletin, 82(4), 463-469. DOI

Alessandri, J., \& Cançado, C. R. (2017). The effects of instructions on the sensitivity of negatively reinforced human behavior to extinction. Behavioural Processes, 136, 50-53. $\underline{\mathrm{DOI}}$

American Psychiatric Association. (2013). Diagnostic and statistical manual of mental disorders $\left(D S M-5^{\oplus}\right)$ : American Psychiatric Pub.

Bateson, M., \& Kacelnik, A. (1996). Rate currencies and the foraging starling: the fallacy of the averages revisited. Behavioral Ecology, 7(3), 341-352. DOI

Berger, D. F., \& Sagvolden, T. (1998). Sex differences in operant discrimination behaviour in an animal model of attention-deficit hyperactivity disorder. Behavioural brain research, 94(1), 73-82. $\underline{\mathrm{DOI}}$

Bickel, W. K., MacKillop, J., Madden, G. J., Odum, A. L., \& Yi, R. (2015). Experimental manipulations of delay discounting \& related processes: An introduction to the special issue. Journal of the Experimental Analysis of Behavior, 103(1), 1-9. DOI

Bizot, J.-C., Chenault, N., Houzé, B., Herpin, A., David, S., Pothion, S., \& Trovero, F. (2007). Methylphenidate reduces impulsive behaviour in juvenile Wistar rats, but not in adult Wistar, SHR and WKY rats. Psychopharmacology, 193(2), 215-223. DOI

Blanchard, T. C., Pearson, J. M., \& Hayden, B. Y. (2013). Postreward delays and systematic biases in measures of animal temporal discounting. Proceedings of the National Academy of Sciences, 110(38), 15491-15496. DOI

Botanas, C. J., Lee, H., de la Peña, J. B., dela Peña, I. J., Woo, T., Kim, H. J., Han, D.H., Kim, B., \& Cheong, J. H. (2016). Rearing in an enriched environment attenuated hyperactivity and inattention in the spontaneously hypertensive rats, an animal model of attention-deficit hyperactivity disorder. Physiology \& Behavior, 155, 30-37. DOI

Cournoyer, M., \& Trudel, M. (1991). Behavioral correlates of self-control at 33 months. Infant Behavior and Development, 14(4), 497-503. DOI

Dalen, L., Sonuga-Barke, E. J., Hall, M., \& Remington, B. (2004). Inhibitory deficits, delay aversion and preschool AD/HD: implications for the dual pathway model. Neural Plasticity, 11(1-2), 1-11. DOI

Dalley, J. W., Everitt, B. J., \& Robbins, T. W. (2011). Impulsivity, compulsivity, and top-down cognitive control. Neuron, 69(4), 680-694. DOI 
Eisenberger, R., \& Masterson, F. A. (1986). Effects of prior learning and current motivation on self-control. Quantitative analyses of behavior, 5, 267-282.

Elner, R. W., \& Hughes, R. N. (1978). Energy maximization in the diet of the shore crab, Carcinus maenas. The Journal of Animal Ecology, 47(1), 103-116. DOI

Fox, A. T., Hand, D. J., \& Reilly, M. P. (2008). Impulsive choice in a rodent model of attentiondeficit/hyperactivity disorder. Behavioural Brain Research, 187(1), 146-152. DOI

Garcia, A., \& Kirkpatrick, K. (2013). Impulsive choice behavior in four strains of rats: Evaluation of possible models of Attention Deficit/Hyperactivity Disorder. Behavioural Brain Research, 238, 10-22. DOI

Goldshmidt, J. N., Lattal, K. M., \& Fantino, E. (1998). Context effects on choice. Journal of the Experimental Analysis of Behavior, 70(3), 301-320. DOI

Green, L., Myerson, J., \& Ostaszewski, P. (1999). Amount of reward has opposite effects on the discounting of delayed and probabilistic outcomes. Journal of Experimental PsychologyLearning Memory and Cognition, 25(2), 418-427. DOI

Hand, D. J., Fox, A. T., \& Reilly, M. P. (2009). Differential effects of d-amphetamine on impulsive choice in spontaneously hypertensive and Wistar-Kyoto rats. Behavioural Pharmacology, 20(5-6), 549-553. DOI

Hayden, B. Y. (2016). Time discounting and time preference in animals: a critical review. Psychonomic Bulletin \& Review, 23(1), 39-53. DOI

Holth, P. (2001). The Persistence of Category Mistakes in Psychology. Behavior and Philosophy, 29, 203-219.

Ibias, J., \& Pellón, R. (2011). Schedule-induced polydipsia in the spontaneously hypertensive rat and its relation to impulsive behaviour. Behavioural Brain Research, 223(1), 58-69. DOI

Íbias, J., \& Pellón, R. (2014). Different relations between schedule-induced polydipsia and impulsive behaviour in the Spontaneously Hypertensive Rat and in high impulsive Wistar rats: Questioning the role of impulsivity in adjunctive behaviour. Behavioural Brain Research, 271, 184-194. DOI

Jeschke, J. M., Kopp, M., \& Tollrian, R. (2002). Predator functional responses: discriminating between handling and digesting prey. Ecological Monographs, 72(1), 95-112. DOI

Johansen, E. B., Killeen, P. R., \& Sagvolden, T. (2007). Behavioral variability, elimination of responses, and delay-of-reinforcement gradients in SHR and WKY rats. Behavioral and Brain Functions, 3(1), 60. DOI

Killeen, P. R. (2015). Models of ADHD: Five ways smaller sooner is better. Journal of Neuroscience Methods, 252, 2-13. DOI

Luman, M., Van Meel, C. S., Oosterlaan, J., Sergeant, J. A., \& Geurts, H. M. (2009). Does reward frequency or magnitude drive reinforcement-learning in attention-deficit/hyperactivity disorder? Psychiatry Research, 168(3), 222-229. DOI

MacArthur, R. H., \& Pianka, E. R. (1966). On optimal use of a patchy environment. The American Naturalist, 100(916), 603-609. DOI

Malloy-Diniz, L., Fuentes, D., Leite, W. B., Correa, H., \& Bechara, A. (2007). Impulsive behavior in adults with attention deficit/hyperactivity disorder: characterization of attentional, motor and cognitive impulsiveness. Journal of the International Neuropsychological Society, 13(4), 693-698. DOI

Marco, R., Miranda, A., Schlotz, W., Melia, A., Mulligan, A., Müller, U., .. Sonuga-Barke, E. (2009). Delay and Reward Choice in ADHD: An Experimental Test of the Role of Delay Aversion. Neuropsychology, 23(3), 367. DOI

Matthews, B. A., Shimoff, E., Catania, A. C., \& Sagvolden, T. (1977). Uninstructed Human Reponding: Sensitivity to Ratio and Interval Contigencies. Journal of the Experimental Analysis of Behavior, 27(3), 453-467. 
Mazur, J. E. (1987). An adjusting procedure for studying delayed reinforcement. In M. Commons, J. Mazur, J. Nevin, \& H. Rachlin (Eds.), Quantitative Analyses of Behavior Volume V: The Effect of Delay and Intervening Events on Reinforcement Value (pp. 55-73). New Jersey: Lawrence Erlbaum Associates Inc.

Odum, A. L. (2011). Delay discounting: I'm ak, you're ak. Journal of the Experimental Analysis of Behavior, 96(3), 427-439. DOI

Orduña, V. (2015). Impulsivity and sensitivity to amount and delay of reinforcement in an animal model of ADHD. Behavioural Brain Research, 294, 62-71. DOI

Pardey, M. C., Homewood, J., Taylor, A., \& Cornish, J. L. (2009). Re-evaluation of an animal model for ADHD using a free-operant choice task. Journal of Neuroscience Methods, 176(2), 166-171. DOI

Pearson, J. M., Hayden, B. Y., \& Platt, M. L. (2010). Explicit information reduces discounting behavior in monkeys. Frontiers in Psychology, 1. DOI

Pierce, G., \& Ollason, J. (1987). Eight reasons why optimal foraging theory is a complete waste of time. Oikos, 49(1), 111-118. DOI

Pyke, G. H. (1978). Optimal foraging in hummingbirds: testing the marginal value theorem. American Zoologist, 18(4), 739-752. DOI

Rachlin, H. (1974). Self-control. Behaviorism, 2(1), 94-107.

Reynolds, B., De Wit, H., \& Richards, J. B. (2002). Delay of gratification and delay discounting in rats. Behavioural Processes, 59(3), 157-168. DOI

Robbins, T. W., \& Everitt, B. J. (1996). Neurobehavioural mechanisms of reward and motivation. Current Opinion in Neurobiology, 6(2), 228-236. DOI

Rosati, A. G., Stevens, J. R., Hare, B., \& Hauser, M. D. (2007). The evolutionary origins of human patience: temporal preferences in chimpanzees, bonobos, and human adults. Current Biology, 17(19), 1663-1668. DOI

Sagvolden, T. (2000). Behavioral validation of the spontaneously hypertensive rat (SHR) as an animal model of attention-deficit/hyperactivity disorder (AD/HD). Neuroscience \& Biobehavioral Reviews, 24(1), 31-39. DOI

Sagvolden, T., Aase, H., Zeiner, P., \& Berger, D. (1998). Altered reinforcement mechanisms in attention-deficit/hyperactivity disorder. Behavioural Brain Research, 94(1), 61-71. DOI

Sagvolden, T., Johansen, E. B., Aase, H., \& Russell, V. A. (2005). A dynamic developmental theory of attention-deficit/hyperactivity disorder (ADHD) predominantly hyperactive/impulsive and combined subtypes. Behavioral and Brain Sciences, 28(3), 397-418. DOI

Scheres, A., Lee, A., \& Sumiya, M. (2008). Temporal reward discounting and ADHD: task and symptom specific effects. Journal of Neural Transmission, 115(2), 221-226. DOI

Sjoberg, E. A. (2017). Logical fallacies in animal model research. Behavioral and Brain Functions, 13(1). DOI

Sjoberg, E. A., Holth, P., \& Johansen, E. B. (2016). The effect of delay, utility, and magnitude on delay discounting in an animal model of Attention-Deficit/Hyperactivity Disorder (ADHD): a systematic review. Paper presented at the Association of Behavior Analysis International 42nd Annual Convention, Chicago, IL. DOI

Sjoberg, E. A., Holth, P., Ottåsen, H. M., \& Johansen, E. B. (2017). The problem with the intertrial-interval in an animal model of ADHD. Paper presented at the Norwegian Behavior Analysis Conference, Storefjell, Norway. DOI

Smethells, J. R., \& Reilly, M. P. (2015). Intertrial interval duration and impulsive choice. Journal of the Experimental Analysis of Behavior, 103(1), 153-165. DOI 
Sonuga-Barke, E., Taylor, E., Sembi, S., \& Smith, J. (1992). Hyperactivity and delay aversion-I. The effect of delay on choice. Journal of Child Psychology and Psychiatry, 33(2), 387-398. $\underline{\mathrm{DOI}}$

Strandberg, R., Klaassen, R. H., Hake, M., Olofsson, P., \& Alerstam, T. (2009). Converging migration routes of Eurasian hobbies Falco subbuteo crossing the African equatorial rain forest. Proceedings of the Royal Society of London B: Biological Sciences, 276(1657), 727-733. DOI

Sundbø, S. M. (2013). Effects of increasing, decreasing and constant delays of reinforcement on choice behavior in rats. (MA in Learning in Complex Systems-Behavioral Analysis), Oslo and Akershus University College of Applied Sciences, Oslo.

Sutherland, K. R., Alsop, B., McNaughton, N., Hyland, B. I., Tripp, G., \& Wickens, J. R. (2009). Sensitivity to delay of reinforcement in two animal models of attention deficit hyperactivity disorder (ADHD). Behavioural Brain Research, 205(2), 372-376. DOI

Tripp, G., \& Alsop, B. (1999). Sensitivity to reward frequency in boys with attention deficit hyperactivity disorder. Journal of Clinical Child Psychology, 28(3), 366-375. DOI

Werner, E. E., \& Hall, D. J. (1974). Optimal foraging and the size selection of prey by the bluegill sunfish (Lepomis macrochirus). Ecology, 55(5), 1042-1052. DOI

White, N. M. (1989). Reward or reinforcement: what's the difference? Neuroscience \& Biobehavioral Reviews, 13(2), 181-186. DOI

Wolff, M. C., \& Leander, J. D. (2002). Selective serotonin reuptake inhibitors decrease impulsive behavior as measured by an adjusting delay procedure in the pigeon. Neuropsychopharmacology, 27(3), 421. DOI

Wooters, T. E., \& Bardo, M. T. (2011). Methylphenidate and fluphenazine, but not amphetamine, differentially affect impulsive choice in Spontaneously Hypertensive, Wistar-Kyoto and Sprague-Dawley rats. Brain Research, 1396, 45-53. DOI 